\title{
See-coast: polarimetric and spectral characterization of exoplanets with a small space telescope
}

\author{
Raphaël Galicher $^{a, c}$ Pierre Baudoz ${ }^{b, c}$ Anthony Boccaletti ${ }^{b, c}$ Anne-Lise Maire ${ }^{b, c}$ \\ Samuel Ronayette ${ }^{d}$ Jean Schneider $^{a}$ and the See-coast team \\ ${ }^{a}$ Luth, CNRS, Observatoire de Paris, 5, place Jules Janssen, 92195 Meudon, France \\ ${ }^{b}$ Lesia, CNRS, Observatoire de Paris, 5, place Jules Janssen, 92195 Meudon, France \\ ${ }^{c}$ Groupement d'Intérêt Scientifique Phase (Partenariat Haute résolution Angulaire Sol Espace) \\ $d$ CEA Saclay (France)
}

\begin{abstract}
To characterize orbits and atmospheres of exoplanets with large orbits ( $\geq$ a few $\mathrm{AU})$, direct imaging is nowadays the sole way. From space, this involves high contrast imaging techniques as coronagraphy, differential imaging or wavefront control. Several methods exist or are under development and several small $(\sim 1.5 \mathrm{~m})$ space telescope missions are proposed. One of them is See-coast (super-Earth explorer coronagraphic off-axis space telescope) which will be proposed to the next ESA Cosmic Vision call. It will provide polarimetric and spectral characterization of giant gazeous planets and possibly Super-Earths in visible light. In this paper, we first detail science cases of this mission. We then describe the foreseen telescope design and its instrumentation. We finally derive performance for a particular instrumental configuration from numerical simulation and we show how See-coast can retrieve planet spectra.
\end{abstract}

Keywords: Instrumentation, High contrast imaging, High angular resolution, exoplanet, wavefront correction, dark hole

\section{INTRODUCTION}

Since $1995,{ }^{1}$ more than 450 exoplanets of different kinds have been discovered while orbiting different types of stars (age, metallicity, position in the Galaxy). One determined their orbital parameters and their radius and thus, by statistics studies, put constraints on models of planetary formation and evolution.$^{2-4}$ In a few cases, spectral data provided informations on the chemical compositions and dynamics of exoplanetary atmospheres..$^{5,6}$ All these studies, still in progress, are directly derived from radial velocity and transit detections, which means that they concern planets orbiting quite close to their star (less than a few astronomical units). To put stronger constraints on formation and evolution models and on atmosphere models, it would be very useful to characterize planets orbiting further to their star. The sole current solution is the direct imaging. Several near-infrared (NIR) ground-based instruments as Sphere [7, VLT], GPI [8, Gemini] and HiCIAO [9, Subaru], are to be implemented on the $8 \mathrm{~m}$ class telescopes in a few years. The aims of these extreme AO systems is the search, and the orbital and spectral characterizations of young and massive planets in the solar neighborhood. As for the space, near and mid infrared spectral characterizations of even lighter and fainter objects such as mature massive planets (1-5 Gyr) on 5 to $10 \mathrm{AU}$ orbits may be done by the Miri instrument of JWST ${ }^{10}$ On the long term (about 2020), the extremely large telescopes (ELT) will step further by providing near-infrared spectral informations and visible polarimetric characterizations about mature massive planets but Super-Earths in ideal conditions.$^{11}$ Finally, the very long term large space missions will characterize by spectro-polarimetry Earth-like planets. But it is now mostly admitted that this will require previous mission(s) as precursor(s). In this context, our team will submit a proposal to the next ESA Cosmic Vision call. We will propose the See-coast space mission concept to characterize giant gazeous planets and possibly Super-Earths both in spectroscopy and polarimetry at visible wavelengths. In this paper, we present the objectives of the See-coast mission in section 2. Section 3 is about the foreseen telescope design and its instrumentation. Section 4 provides expected precisions on measurement of spectral features in a Jupiter-like planet spectrum.

E-mail: raphael.galicher@obspm.fr, Phone number: +33 (0)1 45077617

Space Telescopes and Instrumentation 2010: Optical, Infrared, and Millimeter Wave, edited by Jacobus M. Oschmann Jr., Mark C. Clampin, Howard A. MacEwen, Proc. of SPIE Vol. 7731, 773126 - (c) 2010 SPIE - CCC code: 0277-786X/10/\$18 - doi: 10.1117/12.857880 


\section{SCIENCE CASES AND OBSERVING STRATEGY}

\subsection{Science cases}

\subsubsection{Targets}

The See-coast mission aims to measure the starlight reflected by exoplanets in visible light from $0.4 \mu \mathrm{m}$ to $0.95 \mu \mathrm{m}$ with a spectral resolution between 30 to 100. Targets will be of several kinds: gazeous planets at intermediate semi-major axis (1 to $5 \mathrm{AU}$ ) around nearby stars (closer to a few tens of parsecs) with an expected diversity of atmospheric chemical compositions; Super-Earths - 1.5 to 2.5 larger than Earth but presenting a similar structure with a solid core and a not-dominating atmosphere ${ }^{12}$ - at a few AU around nearby stars; multiple planets in a sole system; exozodiacal disks.

\subsubsection{Determined parameters}

It is possible to estimate several parameters characterizing the planet from direct imaging:

- Orbital parameters: observing the planet at different epochs provides the semi-major axis, excentricity and inclination angle to the line of sight.

- Mass: as the inclination angle is determined, if radial velocity is also used, the exact mass is known.

- Chemical composition of the atmosphere: spectral features of water, oxygen, ozone, ammonia, and methane exist at visible wavelengths. Both Jupiter and Earth-like atmospheres can then be characterized. ${ }^{13,14}$

- Physics of the atmospheres: the column density of clear atmosphere above clouds is derived from the Rayleigh scattering and the polarization ratio of reflected light is a tracer of clouds coverage and haze. ${ }^{14}$

- Surface: oceans and continents have different temperatures, albedos and polarization signatures. ${ }^{14}$

- Duration of the planetary day: when the planet rotates, the temperature, albedo and polarization are modulated, which let us retrieve the planetary day period. This parameter puts strong constraints on planetary formation models.

\subsection{Observing strategy}

The See-coast main objective is the spectro-polarimetric characterization of faint objects and we will have to spend time on each target. That is why the purpose of the mission is not, primarily, to search for new planets, which is time consuming, but to observe planets previously discovered by astrometry or radial velocity surveys. Each target will be visited several times at different epochs (intervals of several months) to constrain orbits and to detect variations on spectral and polarimetric signatures.

We propose to place the telescope at the L2 Sun-Earth Lagragian point so that it will be very stable (cf. requirements in section 3): the temperature fluctuations are minimized and very long continuous exposure can be done (several days and maybe weeks). An other solution would be a drift-away heliocentric orbit combined with an internal thermal control system. The second solution is less combustible consuming but the mission life is restricted to about five years.

\section{TELESCOPE DESIGN AND INSTRUMENTATION}

\subsection{Technical requirements}

Direct imaging of close-in planets ( $1 \mathrm{AU}$ up to $5 \mathrm{AU}$ ) at distances between 3 to 15 parsec requires an angular resolution of about 70 mas and a field of view of about $3 \times 3 \operatorname{arcsec}^{2}$. A strong attenuation of the stellar light is also needed because it is between $\sim 10^{4}$ and $10^{9}$ brighter than its planets. We propose to use a coronagraph. Such a device is very sensitive to central obscuration and we choose a very stable off-axis telescope. A spectral resolution of at least 40 in the visible range $(0.4$ to $0.95 \mu \mathrm{m})$ is needed to well-characterize the chemical composition of the planet. The polarization has to be measured with a $10 \%$ accuracy to determine physics parameters of the atmospheres. Other requirements are detailed in a previous paper. ${ }^{12}$ 


\subsection{Telescope design}

We choose a simple and compact telescope and spacecraft minimizing the number of optics and their optical aberrations. One possible design is proposed in figure 1. Two off-axis parabolic mirrors are combined to provide a $F / 60$ focal plane where the instrumentation is placed. The field of view is $30 \times 30 \operatorname{arcsec}^{2}$. The diameter of the collector surface is $1.5 \mathrm{~m}$ and the telescope is enclosed in a cylinder of $1.7 \mathrm{~m}$ diameter and $3.7 \mathrm{~m}$ length. Optical aberrations (with perfect optics) are smaller than $\lambda / 125 \mathrm{rms}$. Other designs are possible to have a more compact telescope or to enhance the field of view but the wavefront error (with perfect optics) is then slightly increased to $\sim \lambda / 100$. The primary mirror size can also be enlarged in function of cost.

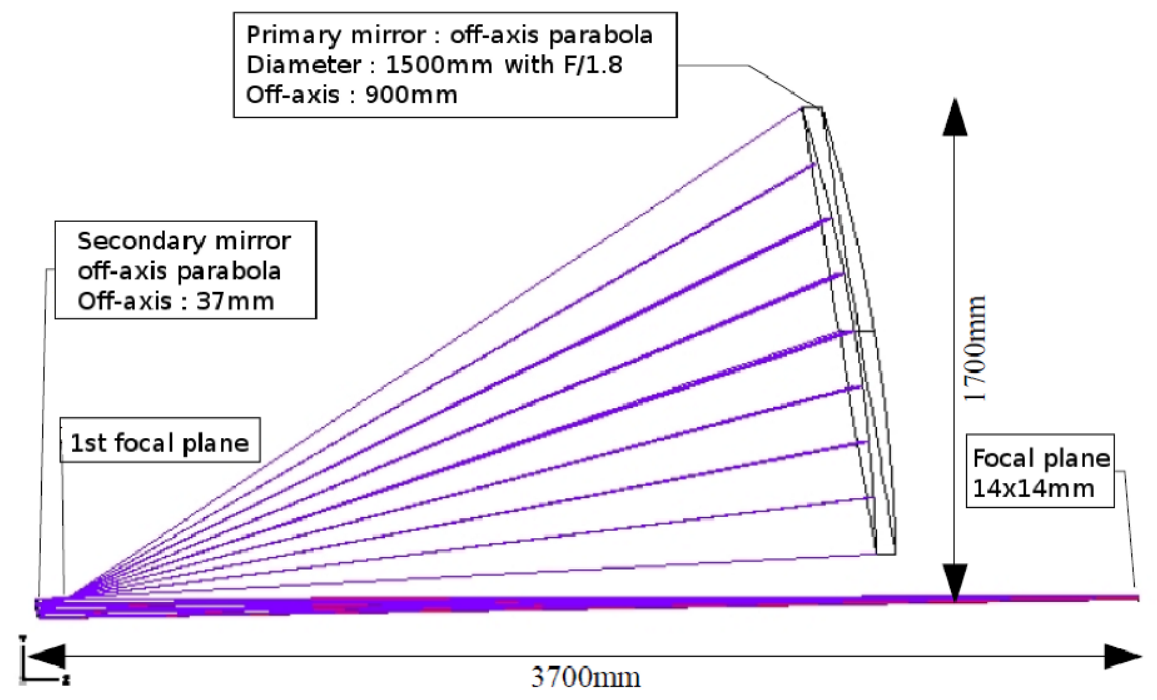

Figure 1. A possible telescope design for the See-coast mission. The primary mirror is a 1.5 m diameter off-axis parabola. The field of view is $30 \times 30$ arcsec $^{2}$ and the final focal plane ratio is $F / 60$.

\subsection{Instrumental configuration}

As the telescope design is chosen as simple as possible, the sole focal intrumentation has to achieve the high contrast imaging. Several techniques are involved in addition to an accurate control of the temperature: stabilization of the pointing, active wavefront correction for quasi-static aberrations, coronagraphy, and speckle suppression module (figure 2). A previous paper ${ }^{12}$ makes a complete description of the See-coast instrumental

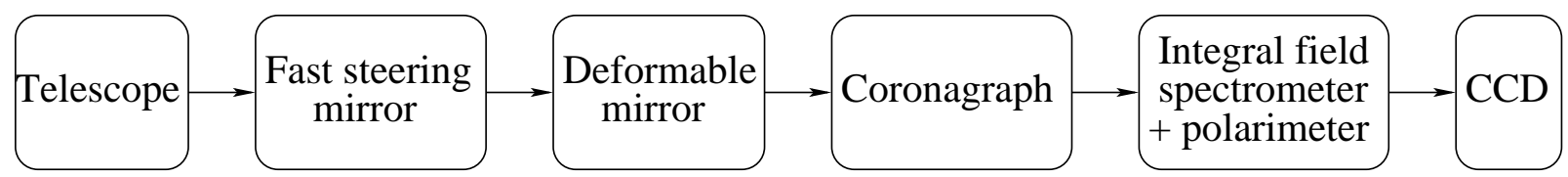

Figure 2. Block diagram of the See-coast payload.

configuration and we encourage the reader to refer to it. Here, we briefly remind some details we need further in the paper.

A coronagraph reduces the stellar flux without affecting the planet image. We envisage focal phase mask coronagraphs so that the inner working angle $(\sim 1$ to $2 \lambda / D)$ is small enough to characterize very-close-to-their-star planets. We will certainly choose between a four quadrant phase mask $[15, \mathrm{FQPM}]$ and an annular groove phase mask [16, AGPM]. In order to minimize wavefront aberrations that strongly limit the coronagraph performance by inducing speckles on the detector, we foresee two options: $1 /$ using a super-polished telescope (less than $5 \mathrm{~nm}$ rms of aberrations up to $\sim 40$ cycles per pupil) or $2 /$ combining a less well-polished telescope $(20 \mathrm{~nm} \mathrm{rms})$ with 
a deformable mirror of thousand(s) of actuactors. We envisage the use of an integral field spectrometer (IFS) to deliver a spectral data cube of the focal plane image. This data cube is needed to have spectral informations on the planet and it is also very useful to reduce the speckle noise using differential imaging, ${ }^{17}$ spectral deconvolution ${ }^{18}$ or a self-coherent camera. ${ }^{19}$

\section{PERFORMANCE}

A thorough study is needed to define the best instrumental configuration in function of sciences cases. In this section, we present results (section 4.2) obtained considering a particuliar instrumental configuration (section 4.1).

\subsection{Assumptions}

A complete study of the performance for all the potential instrumental configurations and all the kinds of targets is beyond the scope of this paper. We here study a unique stellar system formed by a G0V star at 10 parsec harboring a Jupiter-like planet at 4 AU. Spectra of both objects follow Burrows' models. ${ }^{20}$ For the instrumentation part, we consider a particuliar configuration composed by the off-axis telescope presented in figure 1, a deformable mirror, an achromatic FQPM coronagraph associated with a self-coherent camera ${ }^{19}$ and an IFS. The $1.5 \mathrm{~m}$ primary mirror is affected by $20 \mathrm{~nm}$ rms aberrations with a power spectral density similar to the VLT one ${ }^{21}$ evolving in $f^{-3}$ where $f$ is the spatial frequency. The secondary mirror is aberrated with a similar $20 \mathrm{~nm} \mathrm{rms}$ default and we account for the Fresnel propagation of it because the mirror is not in a pupil plane. We assume no further amplitude aberrations. The instrument works in visible light from 0.5 to $1 \mu \mathrm{m}$. The IFS cuts the whole bandpass in 40 channels of identical spectral widths $(\Delta \lambda=12.5 \mathrm{~nm})$. We assume that IFS does not introduce additional noises even if this point has to be ensure in a future study. In our simulations, the polychromatic image of each channel is the sum of 10 monochromatic images with wavelengths uniformly distributed in the considered channel bandpass. The FQPM coronagraph is achromatic and its transitions are infinitly thin. The Lyot stop diaphragm diameter equals $95 \%$ of the pupil diameter. The deformable mirror has a continuous face sheet supported by $32 \times 32$ actuators arranged in a square pattern of constant spacing. It is located in a plane conjugate to the entrance pupil and the nth-influence function is $\exp \left(\ln (0.15)\left(32\left(\xi-\xi_{n}\right) / D\right)^{2}\right)$ where $\xi$ is the pupil coordinate, $\xi_{n}$ locates the center of the nth-actuator, and $D$ is the pupil diameter. ${ }^{22}$ The estimated aberrated phase is expanded following the method of energy minimization in the pupil plane proposed in a previous paper [21, Eq. A3]. The focal plane corrected area is shrunk by a factor 1.05 for optimization. For speckle calibration, we use a self-coherent camera $^{23,24}$ which spatially modulates the stellar speckles with Fizeau fringes in the detector plane and does not affect the planet image. The SCC requires an additional diaphragm of $D / 20$ diameter in the Lyot stop plane. It is separated by $\sim 1.7 D$ from the classical Lyot stop center. ${ }^{19}$ To have a sufficient spatial sampling of the interferential fringes, the detector has 4 pixels per $\lambda / D$ for the smallest wavelength of the bandpass $(0.5 \mu \mathrm{m})$. No read out noise is accounted for. We assume that we perfectly estimate for wavefront errors. To do so, at the beginning of the mission, we use the SCC focal plane image and reach the deformable mirror limitation. ${ }^{19,25}$ Then, during the mission, to account for slow drifts of the aberrations (mainly thermal variations), adjustments of the deformable mirror shape are done still using the SCC. As these corrections are small, the required time is negligible compared to the exposure time required to record the planet spectra.

\subsection{Results}

We calculate the IFS datacubes (2D images and 40 spectral channels) obtained with the See-coast telescope under the assumptions detailed in the previous section. We will present the results studying images and their radial profils, and the estimated planet spectra.

Figure 3 shows the polychromatic images provided by the four quadrant phase mask (FQPM) coronagraph associated with the deformable mirror before (left) and after (right) speckle calibration by the SCC. The polychromatic FQPM image is the sum of all the IFS channels recorded if no SCC speckle calibration is applied. The polychromatic SCC image is the sum of the 40 SCC post-processed images (and not the post-processing of the FQPM polychromatic image which would be useless for calibrating speckles because of chromatism impact on SCC performance $\left.{ }^{19}\right)$. On both images, the dark hole size $\left(32 \times 32\left(\lambda_{0} / D\right)^{2}\right)$ is set by the deformable mirror number of actuators (finite number of corrected spatial frequencies in the pupil plane). Under assumptions of section 4.1, using a sole FQPM with the deformable mirror (image on the left) is not sufficient to detect the 

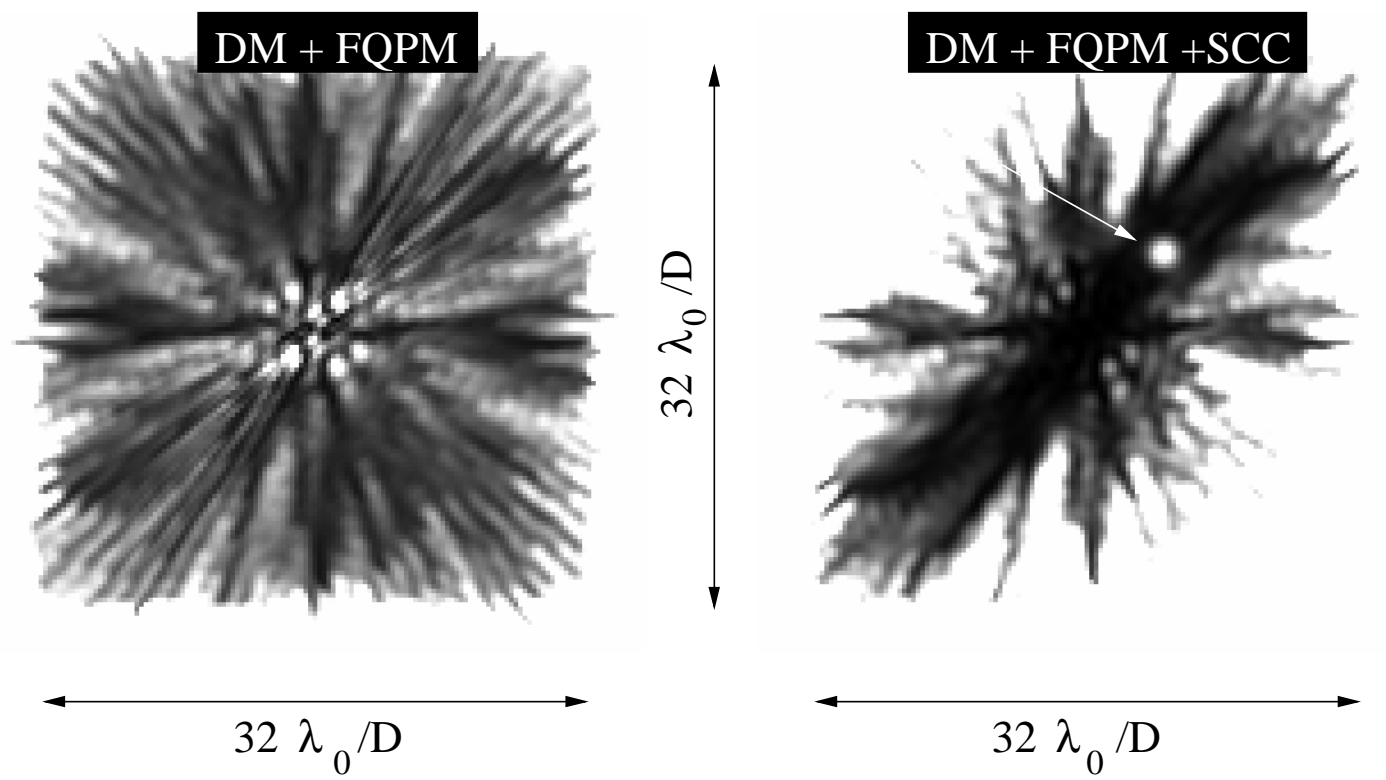

Figure 3. Polychromatic images provided by the See-coast instrumentation (FQPM coronagraph plus deformable mirror) before (left) and after (right) speckle calibration. Scales are linear but different: contrast in white areas in the SCC image is 20 time better than in the FQPM one. The darker the image, the better the contrast.

Jupiter-like planet because stellar speckles still dominate the recorded image (wings of the uncorrected speckles outside the dark hole). The self-coherent camera calibrates these residuals and the planet is detected (arrow on the right). In the SCC image, a direction (from bottom left to top right) is slightly favoured (about a factor 10 in intensity) because the channel chromatism (12.5 nm bandwidth) impacts the SCC post-processing. ${ }^{19}$

The figure 4 plots the radial profiles of the $5 \sigma$ detection in images recorded by the detector after the FQPMDM association before (green full line) and after (red dashed line) the SCC speckle calibration for an infinite exposure time (no photon noise, left) and for an exposure of $10 \mathrm{~h}$ (right). Profiles are also plotted for off-axis star (no coronagraph case, black dot-dashed lines). In all cases, we represents the $5 \sigma$ detection profil of each image of the 40 IFS channels. For each curve, the horizontal axis is graduated in $\lambda / D$ with $\lambda$ the central wavelength of the considered channel. The horizontal axis thus represents the spatial frequencies of the pupil plane: all curves show the DM cut-off at $16 \lambda / D$. We normalize all the curves by $\left(\lambda / \lambda_{0}\right)^{4}$ to account for both the PSF size and the flux $\left(1 / \lambda^{2}\right.$ factor in the focal plane intensity expression, not accounting for the spectrum shape) dependences in $\lambda$ ( $\lambda_{0}$ is the central wavelength of the whole bandpass, $750 \mathrm{~nm}$ here). We adopt these two scaling to get plots easier to interpret.

On the left, we do not account for photon noise (infinite exposure time). All curves are well superimposed and are smooth, which means that performance does not depend on wavelength. To effectively get such performance, an achromatic coronagraph (for exemple a multi-FQPM ${ }^{26}$ ) is required and amplitude errors have to be minimized. Before calibration (green curves), the contrast is strongly enhanced in the dark hole (darker area seen in the image in figure 3). The SCC calibration provides even better contrasts mainly for separations smaller than $10 \lambda / D$. The calibration is not very effective for larger separations because Fizeau fringes on the stellar speckles are blurred due to chromatism of individual channel ( $12.5 \mathrm{~nm}$ bandpass) and the spatial modulation vanishes. The result is however attractive because companions that are about $10^{-10}$ less brighter to their star can be detected at very small separations. An other technique that may help to get better contrasts at large separations $(>10 \lambda / D)$ is the spectral deconvolution. ${ }^{18}$ But this technique is limited by Fresnel propagation effects and optics have to be placed as close as possible to planes conjugate to the entrance pupil plane. Under the assumptions we make in this simulation, the spectral deconvolution does not improve the image quality (because of Fresnel propagation of the secondary mirror). 

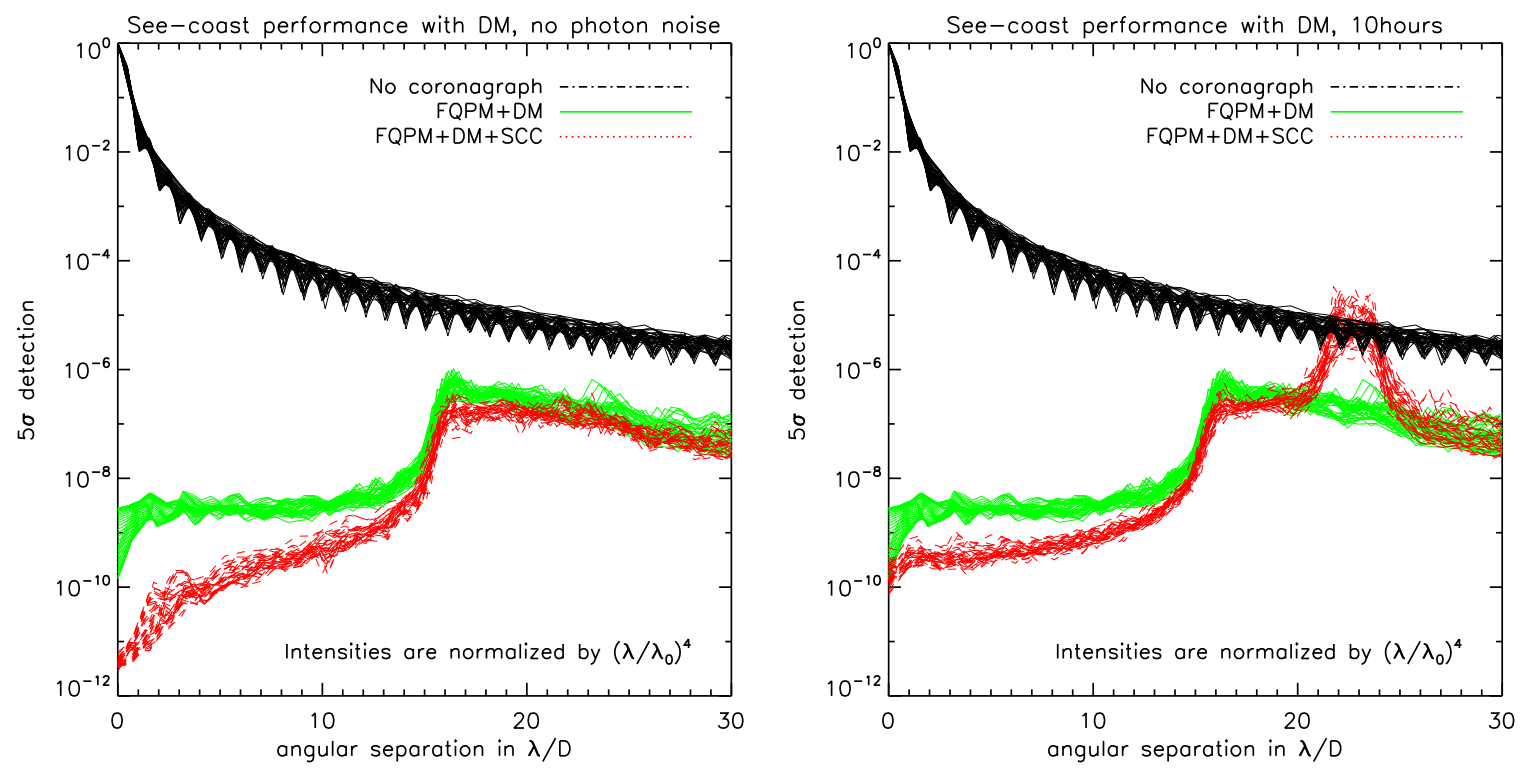

Figure 4. Radial profiles of the $5 \sigma$ detection in images recorded by the detector after the FQPM-DM association before (green full line) and after (red dashed line) the SCC speckle calibration. Profiles are also plotted for off-axis star (no coronagraph case, black dot-dashed lines). In all cases, we represents the $5 \sigma$ detection profil of each image of the 40 IFS channels. The horizontal axis represents the spatial frequencies of the pupil plane (scaling by $\lambda / \lambda_{\min }$, with $\left.\lambda_{\text {min }}=0.5 \mu m\right)$. Curves are normalized by $\left(\lambda / \lambda_{0}\right)^{4}$ to account for both the PSF size and the flux dependences in $\lambda\left(\lambda_{0}=750 \mathrm{~nm}\right)$. We adopt these two scaling to get plots easier to interpret.

For a $10 \mathrm{~h}$ exposure time (right plot), the coronagraphic rejection (green curves) is almost the same than without photon noise. On the contrary, the SCC speckle calibration is less effective and can even deteriorate the performance at some separations (bump at $\sim 24 \lambda / D$, see papers on the self-coherent camera for precise explanations $\left.{ }^{19,25}\right)$. It then exists a minimum exposure time so that photon noise does not dramaticaly impact on the speckle calibration.
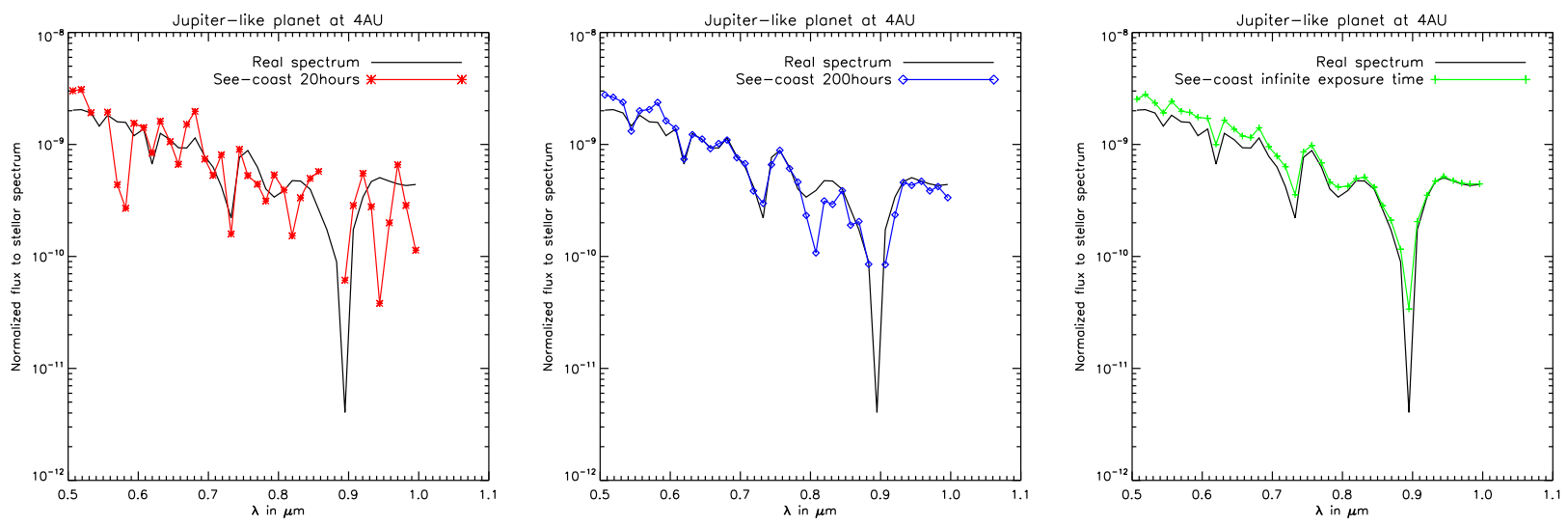

Figure 5. Estimated spectra from the FQPM-DM-SCC images provided by the See-coast telescope after 20 $h$ (left), $200 \mathrm{~h}$ (center) and an infinite (right) exposure time. The full black line reminds the real Jupiter-like planet spectrum. Lines at $0.62 \mu \mathrm{m}, 0.74 \mu \mathrm{m}$, and $0.89 \mu \mathrm{m}$ correspond to methane features.

The detection of the Jupiter-like planet we consider in this simulation (at $4 \mathrm{AU}$ around a G0V) can be done in 10 hours with the DM-FQPM-SCC intrumentation. The image is similar to the one shown on the right in figure 3 but with more noise. However, the spectral characterization of this planet requires a longer exposure to overcome the photon noise impact in every IFS channel. The figure 5 presents the planet spectra estimated 
from the datacube of SCC post-processed images for three exposure times: $20 \mathrm{~h}$ (left), $200 \mathrm{~h}$ (center) and an infinite exposure time (right). Each spectrum is composed of 40 measures corresponding to the 40 IFS channels. Integration of the flux is done over a disk centered on the planet position and with a $2 \lambda / D$ radius. The real spectrum of the light reflected by the planet is overplotted in black full line in each plot at the same spectral resolution. After $20 \mathrm{~h}$, the extracted spectrum is quite noisy and only gives a rough approximation of the real spectrum. After $200 \mathrm{~h}$, the main methane features of the spectrum are retrieved at $0.62 \mu \mathrm{m}, 0.74 \mu \mathrm{m}$, and $0.89 \mu \mathrm{m}$. We can expect to ameliorate the accuracy of the feature estimation by increasing the exposure time because without photon noise, the retrieval spectrum is very close to the real one.

\section{CONCLUSIONS}

The See-coast mission is designed to provide orbital parameter estimations and spectral and polarization characterizations of Jupiter-like planets and super-Earths in visible light. Such a mission would be complementary of ohter ground and space instruments dedicated to direct imaging of exoplanets in near and mid infrared. In this paper, we gave the expected performance for a particular instrumental configuration involving a $1.5 \mathrm{~m}$ off-axis telescope, a deformable mirror, a phase mask coronagraph, and post-coronagraphic technique to calibrate residual speckles. Methane features in the spectrum of Jupiter-like planet orbiting at $4 \mathrm{AU}$ around a G0V at $10 \mathrm{pc}$ are well retrieved. In future works, we will study performance of other instrumental configurations to minimize exposure times needed to get spectral information and we will also consider different kinds of stars and planets. We will submit the final project to the next ESA Cosmic Vision calling in late 2010.

\section{REFERENCES}

[1] Mayor, M. and Queloz, D., "A jupiter-mass companion to a solar-type star," Nature 378, 355-+ (Nov. 1995).

[2] Santos, N. C., "Extra-solar planets: Detection methods and results," New Astronomy Review 52, 154-166 (June 2008).

[3] Cumming, A., Butler, R. P., Marcy, G. W., Vogt, S. S., Wright, J. T., and Fischer, D. A., "The keck planet search: Detectability and the minimum mass and orbital period distribution of extrasolar planets," Publications of the Astronomical Society of the Pacific 120, 531-554 (May 2008).

[4] Marcy, G. W., Butler, R. P., Vogt, S. S., Fischer, D. A., Wright, J. T., Johnson, J. A., Tinney, C. G., Jones, H. R. A., Carter, B. D., Bailey, J., O’Toole, S. J., and Upadhyay, S., "Exoplanet properties from Lick, Keck and AAT," Physica Scripta Volume T 130, 014001-+ (Aug. 2008).

[5] Tinetti, G., Deroo, P., Swain, M. R., Griffith, C. A., Vasisht, G., Brown, L. R., Burke, C., and McCullough, P., "Probing the Terminator Region Atmosphere of the Hot-Jupiter XO-1b with Transmission Spectroscopy," The Astrophysical Journal Letters 712, L139-L142 (Apr. 2010).

[6] Christiansen, J. L., Ballard, S., Charbonneau, D., Madhusudhan, N., Seager, S., Holman, M. J., Wellnitz, D. D., Deming, D., A'Hearn, M. F., and the EPOXI Team, "Studying the Atmosphere of the Exoplanet HAT-P-7b Via Secondary Eclipse Measurements with EPOXI, Spitzer, and Kepler," The Astrophysical Journal 710, 97-104 (Feb. 2010).

[7] Beuzit, J.-L., Feldt, M., Dohlen, K., Mouillet, D., Puget, P., Wildi, F., Abe, L., Antichi, J., Baruffolo, A., Baudoz, P., Boccaletti, A., Carbillet, M., Charton, J., Claudi, R., Downing, M., Fabron, C., Feautrier, P., Fedrigo, E., Fusco, T., Gach, J.-L., Gratton, R., Henning, T., Hubin, N., Joos, F., Kasper, M., Langlois, M., Lenzen, R., Moutou, C., Pavlov, A., Petit, C., Pragt, J., Rabou, P., Rigal, F., Roelfsema, R., Rousset, G., Saisse, M., Schmid, H.-M., Stadler, E., Thalmann, C., Turatto, M., Udry, S., Vakili, F., and Waters, R., "SPHERE: a planet finder instrument for the VLT," in [Society of Photo-Optical Instrumentation Engineers (SPIE) Conference Series], Society of Photo-Optical Instrumentation Engineers (SPIE) Conference Series 7014 (Aug. 2008).

[8] Macintosh, B. A., Graham, J. R., Palmer, D. W., Doyon, R., Dunn, J., Gavel, D. T., Larkin, J., Oppenheimer, B., Saddlemyer, L., Sivaramakrishnan, A., Wallace, J. K., Bauman, B., Erickson, D. A., Marois, C., Poyneer, L. A., and Soummer, R., "The Gemini Planet Imager: from science to design to construction," in [Society of Photo-Optical Instrumentation Engineers (SPIE) Conference Series], Society of Photo-Optical Instrumentation Engineers (SPIE) Conference Series 7015 (July 2008). 
[9] Tamura, M., Hodapp, K., Takami, H., Abe, L., Suto, H., Guyon, O., Jacobson, S., Kandori, R., Morino, J., Murakami, N., Stahlberger, V., Suzuki, R., Tavrov, A., Yamada, H., Nishikawa, J., Ukita, N., Hashimoto, J., Izumiura, H., Hayashi, M., Nakajima, T., and Nishimura, T., "Concept and science of HiCIAO: high contrast instrument for the Subaru next generation adaptive optics," in [Society of Photo-Optical Instrumentation Engineers (SPIE) Conference Series], Society of Photo-Optical Instrumentation Engineers (SPIE) Conference Series 6269 (July 2006).

[10] Boccaletti, A., Baudoz, P., Baudrand, J., Reess, J. M., and Rouan, D., "Imaging exoplanets with the coronagraph of JWST/MIRI," Advances in Space Research 36, 1099-1106 (2005).

[11] Kasper, M. E., Beuzit, J., Verinaud, C., Yaitskova, N., Baudoz, P., Boccaletti, A., Gratton, R. G., Hubin, N., Kerber, F., Roelfsema, R., Schmid, H. M., Thatte, N. A., Dohlen, K., Feldt, M., Venema, L., and Wolf, S., "EPICS: the exoplanet imager for the E-ELT," in [Society of Photo-Optical Instrumentation Engineers (SPIE) Conference Series], Society of Photo-Optical Instrumentation Engineers (SPIE) Conference Series 7015 (July 2008).

[12] Schneider, J., Boccaletti, A., Mawet, D., Baudoz, P., Beuzit, J., Doyon, R., Marley, M., Stam, D., Tinetti, G., Traub, W., Trauger, J., Aylward, A., Cho, J., Keller, C., and Udry, S., "Super earth explorer: a coronagraphic off-axis space telescope," Experimental Astronomy 23, 357-377 (Mar. 2009).

[13] Stam, D. M., Hovenier, J. W., and Waters, L. B. M. F., "Numerical Simulations of the Polarization of Jupiter-like Extrasolar Giant Planets," in [Astronomical Polarimetry: Current Status and Future Directions], A. Adamson, C. Aspin, C. Davis, \& T. Fujiyoshi, ed., Astronomical Society of the Pacific Conference Series 343, 207-+ (Dec. 2005).

[14] Stam, D. M., "Spectropolarimetric signatures of Earth-like extrasolar planets," Astronomy and Astrophysics 482, 989-1007 (May 2008).

[15] Rouan, D., Riaud, P., Boccaletti, A., Clénet, Y., and Labeyrie, A., "The four-quadrant phase-mask coronagraph. i. principle," Publications of the Astronomical Society of the Pacific 112, 1479-1486 (Nov. 2000).

[16] Mawet, D., Riaud, P., Absil, O., and Surdej, J., "Annular groove phase mask coronagraph," The Astrophysical Journal 633, 1191-1200 (Nov. 2005).

[17] Marois, C., Doyon, R., Nadeau, D., Racine, R., Riopel, M., Vallée, P., and Lafrenière, D., "Trident: An infrared differential imaging camera optimized for the detection of methanated substellar companions," Publications of the Astronomical Society of the Pacific 117, 745-756 (July 2005).

[18] Thatte, N., Abuter, R., Tecza, M., Nielsen, E. L., Clarke, F. J., and Close, L. M., "Very high contrast integral field spectroscopy of AB Doradus C: 9-mag contrast at 0.2arcsec without a coronagraph using spectral deconvolution," Monthly Notices of the Royal Astronomical Society 378, 1229-1236 (July 2007).

[19] Galicher, R., Baudoz, P., Rousset, G., Totems, J., and Mas, M., "Self-coherent camera as a focal plane wavefront sensor: simulations," Astronomy and Astrophysics 509, A260000+ (Jan. 2010).

[20] Burrows, A., "A theoretical look at the direct detection of giant planets outside the Solar System," $N a$ ture 433, 261-268 (Jan. 2005).

[21] Bordé, P. J. and Traub, W. A., "High-contrast imaging from space: Speckle nulling in a low-aberration regime," Astrophysical Journal 638, 488-498 (Feb. 2006).

[22] Huang, L., Rao, C., and Jiang, W., "Modified gaussian influence function of deformable mirror actuators," Optical Society of America Journal 16, 108 - 114 (2008).

[23] Baudoz, P., Boccaletti, A., Baudrand, J., and Rouan, D., "The self-coherent camera: a new tool for planet detection," in [IAU Colloq. 200: Direct Imaging of Exoplanets: Science Techniques], Aime, C. and Vakili, F., eds., 553-558 (2006).

[24] Galicher, R. and Baudoz, P., "Expected performance of a self-coherent camera," Comptes Rendus Physique 8, 333-339 (Apr. 2007).

[25] Galicher, R., Baudoz, P., and Rousset, G., "Wavefront error correction and earth-like planet detection by a self-coherent camera in space," Astronomy and Astrophysics 488, L9-L12 (Sept. 2008).

[26] Baudoz, P., Galicher, R., Baudrand, J., and Boccaletti, A., "Theory and laboratory tests of the multistage phase mask coronagraph," in [Society of Photo-Optical Instrumentation Engineers (SPIE) Conference Series], Presented at the Society of Photo-Optical Instrumentation Engineers (SPIE) Conference 7015 (July 2008). 